\title{
BMJ Open Primary care interventions to improve transition of youth with chronic health conditions from paediatric to adult healthcare: a systematic review
}

Jasmin Bhawra, ${ }^{1}$ Alene Toulany, ${ }^{2,3,4}$ Eyal Cohen, ${ }^{1,4,5}$ Charlotte Moore Hepburn, ${ }^{1,4}$ Astrid Guttmann ${ }^{1,3,4,5}$

To cite: Bhawra J, Toulany A, Cohen $\mathrm{E}$, et al. Primary care interventions to improve transition of youth with chronic health conditions from paediatric to adult healthcare: a systematic review. BMJ Open 2016;6: e011871. doi:10.1136/ bmjopen-2016-011871

- Prepublication history and additional material is available. To view please visit the journal (http://dx.doi.org/ 10.1136/bmjopen-2016011871).

$\mathrm{JB}$ and AT contributed equally as co-primary author.

Received 10 March 2016 Revised 28 March 2016 Accepted 13 April 2016

CrossMark

For numbered affiliations see end of article.

Correspondence to Dr Astrid Guttmann; astrid.guttmann@ices.on.ca

\section{ABSTRACT}

Objective: To determine effective interventions to improve primary care provider involvement in transitioning youth with chronic conditions from paediatric to adult care.

Design: Systematic review. Multiple electronic databases were searched including Ovid MEDLINE, EMBASE and Web of Science (from 1 January 1947 to 5 August 2015). Evidence quality was assessed using a 36-point scoring system for disparate study designs.

Setting: Studies with paediatric-to-adult transition programmes and interventions involving primary care providers or in primary care settings.

Participants: Youth aged 16 years and over.

Outcomes: Relevant outcomes were grouped into 3 main domains based on the Triple Aim Framework: experience of care, population health, cost.

Results: A total of 1888 unique citations were identified, yielding 3 studies for inclusion. Overall, primary care provider roles were not well defined. 2 studies used case managers to facilitate referrals to primary care, and the remaining study was the only 1 situated in a primary care setting. None of the studies examined transition in all 3 Triple Aim Framework domains. The most commonly reported outcomes were in the cost domain.

Conclusions: There is limited empiric evidence to guide primary care interventions to improve transition outcomes for youth with chronic conditions. Future research and policy should focus on developing and evaluating coordinated transition interventions to better integrate primary care for high need populations.

\section{INTRODUCTION}

An estimated 15-18\% of youth in North America have a chronic health condition and over $98 \%$ of these youth are expected to reach age 20, thereby requiring transition from paediatric to adult healthcare. ${ }^{1}{ }^{2}$ Although there is no universally accepted age of transfer (one-time event), many jurisdictions use inflexible age cut-offs to

\section{Strengths and limitations of this study}

- This systematic review used a rigorous methodology and was an exhaustive search of all studies of transition interventions involving primary care with no restrictions placed on language, jurisdiction, or disease type.

- The main limitation of this systematic review is the small number of studies (three) that have assessed primary care-based transition interventions even with inclusion criteria that did not mandate randomised trial design. In addition, differences in primary care provision for children across various healthcare jurisdiction makes generalisability of study findings challenging. The only study that was based in a primary care setting was in paediatrician-led US practices which may not be relevant to systems where general practitioners provide 'cradle to grave' primary care.

- The review highlights the need for more rigorous intervention studies to guide improved integration of primary and specialised care during transition and better define the role of primary care providers in facilitating an effective transition for youth with chronic conditions.

delineate service boundaries, creating fragmentation and discontinuity in provision of care across a particularly vulnerable period. $^{3}{ }^{4}$ The transition period encompasses multiple steps in a process including thoughtful planning, the actual transfer from paediatric to adult care, and adjustment to the new system afterwards. As such, it can be challenging for patients, families and clinicians alike. ${ }^{5}$ More than half of the youth with chronic health conditions report inadequate support and services during their transition to adult healthcare. ${ }^{6-9}$ It is well recognised that the process is often flawed due to inadequate planning, poor service coordination, lack of resources and gaps in 
education and training. ${ }^{10-12}$ Transition for youth with chronic health conditions is also complicated by the intricate physical and psychosocial developmental changes that accompany adolescence. ${ }^{3} 1314$ Together, these challenges contribute to potentially serious health-related consequences including patient disengagement, poor treatment adherence, increased hospitalisation rates and overall detrimental health outcomes. ${ }^{10} 15-21$

The goal of healthcare transition for youth with chronic health conditions should be to maximise longterm functioning through the delivery of high-quality, developmentally appropriate healthcare services that continue uninterrupted during the passage from adolescence to adulthood. ${ }^{1422} 23$ To date, the majority of programmes designed to enhance transition for youth with chronic illness have been condition-specific or subspecialty-specific and hospital-based with little focus on primary care. ${ }^{24}{ }^{25}$ Much of the currently available literature is primarily descriptive, focuses on the transfer component (one-time event) of transition (purposeful, planned process) and is limited to only a few studies measuring the effectiveness of any particular transition intervention. ${ }^{45-27}$

Although national bodies have recommended more effective primary care provider involvement during transition and integrated primary and specialised care, ${ }^{24} 2829$ the role of primary care in facilitating transition and potentially mitigating adverse events associated with changes in care for youth with chronic conditions remains unclear. ${ }^{24}{ }^{28}$ In addition to specialty care, paediatric to adult primary care transition may also be needed for youth in health systems that rely heavily on paediatricians for primary care. Few transition programmes base their services in primary care settings and utilise the primary care provider to assess and coordinate the health needs of the adolescent and family. ${ }^{24}$ The aim of this systematic review is to determine effective interventions to improve the role of primary care providers in the transition of youth with chronic health conditions from paediatric to adult healthcare.

\section{METHODS}

\section{Search strategy}

A systematic review of all literature and conference proceedings published in the past 65 years (1 January 1947 to 5 August 2015) was conducted using EMBASE, Web of Science, Ovid 'MEDLINE' and Ovid 'In Process \& Other Non-indexed Citations'. Authors of all relevant conference abstracts were contacted to enquire about publications, as only published articles are included in the final results.

The literature search focused on interventions and evaluations targeting a paediatric population (using terms such as 'youth', 'adolescent' and 'paediatric'), transition (ie, 'continuity of care' and 'transfer') and primary care (ie, 'medical home', 'family practice', 'family physician' and 'paediatrician'). Articles published in all languages and all study designs were included in the search. The search was not limited to specific diseases in order to be inclusive of all chronic medical and mental health conditions (see online supplementary file for full search strategy). Articles discussing opinion-based best practice, those defining family/ caregivers as primary care providers and transfers between hospital services or departments were excluded.

\section{Study selection}

Two reviewers (JB and AT) screened all article titles and abstracts. The articles were first screened independently and then examined in full by both reviewers for the inclusion criteria. All identified non-English papers were in French and were assessed by a bilingual member of the research team (AG). The reviewers met to discuss any disagreements which were resolved by consensus (JB, AT, EC and AG).

\section{Data synthesis and quality analysis}

Data from the final included studies were evaluated using a data abstraction table summarising study characteristics, level of primary care engagement and outcomes as per the Triple Aim Framework domains (experience of care, population health, cost)..$^{30} 31$ The reviewers identified which Triple Aim domains were assessed by each study outcome (table 1). Quality scores were also calculated for each of the articles using Hawker et $a l \mathrm{~s}^{32}$ scoring system for disparate study designs. A 36-point scale based on nine domains (abstract and title, introduction and aims, methods and data, sampling, data analysis, ethics and bias, results, transferability or generalisability, and implications and usefulness) was used to grade each study. Two authors (JB and AT) scored the studies and resolved disputes by consensus.

\section{RESULTS}

\section{Literature overview}

Figure 1 illustrates the results of the search and screening strategies. A total of 1888 unique citations were

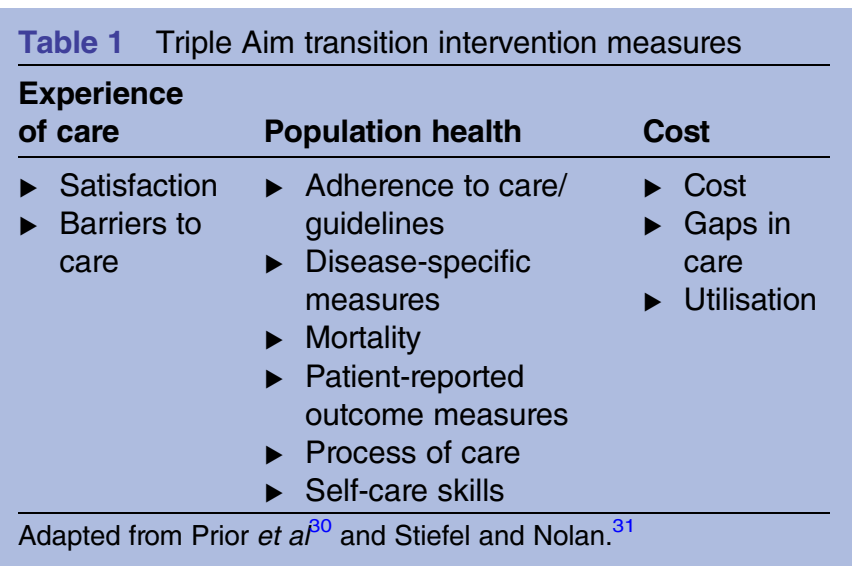




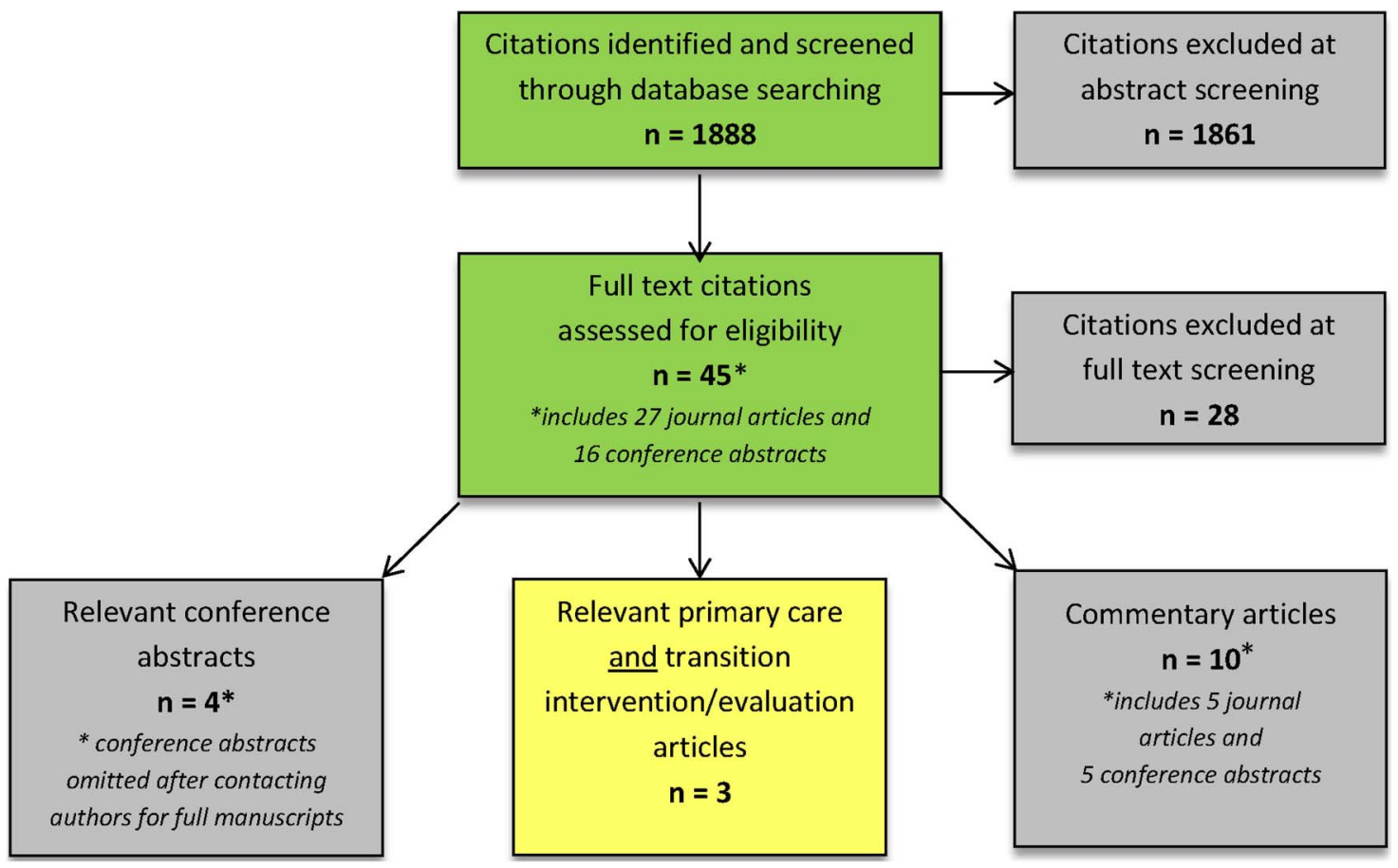

Figure 1 Overview of search results.

identified and screened. In total, 1061 articles were excluded at the abstract screening stage and 45 citations (including 27 journal articles and 16 conference abstracts) were assessed for eligibility. Twenty-seven citations were excluded after full-text review because they did not discuss transition in a primary care setting. Ten of these citations were relevant commentaries on the role of primary care in transition, but were not interventions or evaluations. In total, seven citations discussed transition interventions or evaluations and primary care, including three journal articles ${ }^{33-35}$ and four conference proceeding abstracts, ${ }^{36-39}$ of which only one was eligible. The authors of the abstract were contacted; however, there were no associated article publications of the preliminary results.

\section{Study characteristics}

The three included studies selected were conducted in the USA $(\mathrm{n}=2)^{33}{ }^{34}$ and Canada $(\mathrm{n}=1) .{ }^{35}$ The studies sampled youth with chronic health conditions, ${ }^{34}$ sickle cell disease $(\mathrm{SCD})^{33}$ and type 1 diabetes. ${ }^{35}$ The programme/intervention target groups were most often patients and their families; however, feedback from healthcare providers was an outcome in one of the three studies. ${ }^{33}$ There were no randomised controlled trials in primary care settings or with primary care providers. The three articles included one retrospective cohort, one uncontrolled cohort and a one-time series comparative study. Table 2 describes each study by intervention/ programme, patient condition, location, primary care engagement strategy, study design, outcomes/results and Triple Aim Framework domain addressed. Calculated quality scores for all studies yielded a mean of 26.67 (SD 3.33 ) and median of 25 (range 24.5-30.5). The assessment of risk of bias in individual studies was assessed systematically at the study and outcome levels using the quality score framework described by Hawker et $a \vec{l}^{2}$ for disparate study designs.

\section{Studies}

Hankins $e t a l^{33}$ conducted a retrospective cohort study of a pilot programme aimed at helping adolescents aged 17-19 years with SCD to find an adult medical home. The study assessed 83 adolescents' participation, patient/family/provider acceptance of the programme and number of appointments with adult haematologists fulfilled as an indication of programme efficacy. The programme entailed a tour of adult SCD programmes for patients and families and a lunch with programme staff to discuss transition issues such as expectations, barriers and insurance. A paediatric haematology nurse case manager also scheduled patients' first visit with an adult SCD programme or healthcare provider. This study measured participants' versus non-participants' attendance, completion of a first appointment with an adult healthcare provider, as well as patient/parent/provider satisfaction with the programme using surveys. Primary care was involved to the extent that a case manager helped establish patients' relationship with an adult medical home or healthcare provider.

McManus et $a l \mathrm{~s}^{34}$ time series comparative study involved five health centres which adopted the American Academy of Paediatrics' (AAP) Six Core Elements of Health Care Transition ${ }^{28}$ to assess 


\section{Transition}

intervention

programme

Pilot programme aimed at improving continuity

of care by helping

adolescents identify an

adult medical home.

Consists of 3

components including

a tour of SCD

programmes, lunch

discussion with staff,

and team scheduling

patient's 1st visit to the

adult programme.

Attendance records

consulted and patient/

parent and healthcare

provider feedback

obtained from

surveys. ${ }^{33}$

\section{Academic paediatric} and adult health centre

teams adopted a

2-year learning

collaborative to

implement 'Six Core

elements of Healthcare

Transition'. Teams

consisted of a

physician and a

transition care

coordinator and utilised

the Health Care

Transition Index to

assess programme

progress in

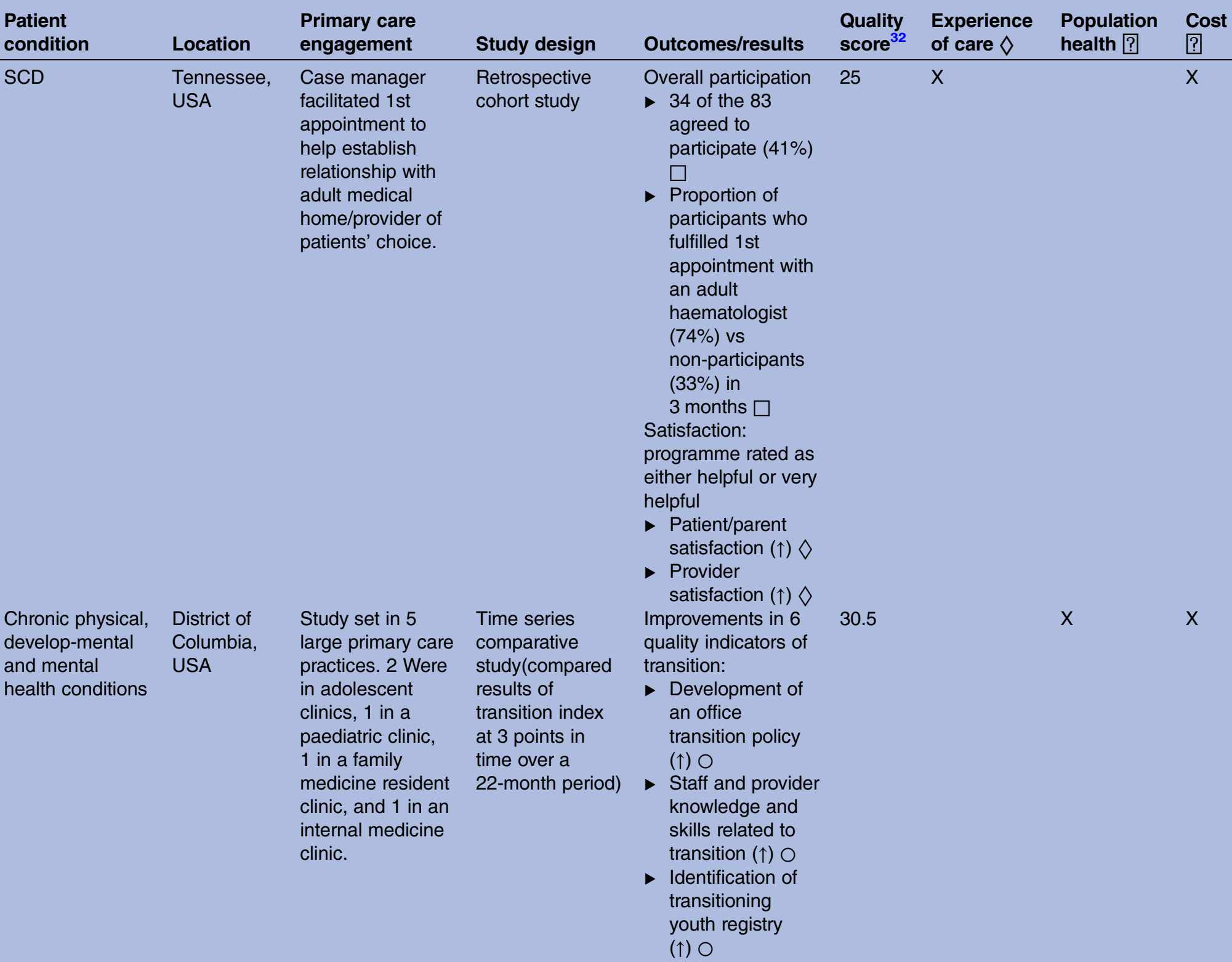

Chronic physical, District of develop-mental Columbia, and mental

USA health conditions

Study set in 5

large primary care

practices. 2 Were

in adolescent

clinics, 1 in a

paediatric clinic,

1 in a family

medicine resident

clinic, and 1 in an

internal medicine

clinic.

Time series

comparative

study(compared

results of

transition index

at 3 points in

time over a

22-month period)

Qutcomes/results Quality Experience

score $^{32}$ of care $\diamond$

Population Cost

Overall participation

25

$\mathrm{X}$
health ?

?

- 34 of the 83

agreed to

participate $(41 \%)$$$
\square
$$

- Proportion of

participants who

fulfilled 1st

appointment with

an adult

haematologist

(74\%) vs

non-participants

(33\%) in

3 months $\square$

Satisfaction:

programme rated as

either helpful or very

helpful

- Patient/parent

satisfaction $(\uparrow) \diamond$

- Provider

satisfaction $(\uparrow) \diamond$

Improvements in 6

quality indicators of

30.5

$x$

X

sition:

- Development of

an office

transition policy

$(\uparrow) \bigcirc$

Staff and provider

knowledge and skills related to

transition $(\uparrow) \bigcirc$

- Identification of

transitioning

youth registry

$(\uparrow) \bigcirc$ 
Triple Aim domains

\section{Transition}

intervention/

Patient

programme

condition

Location

Primary care

engagement

Study design

Outcomes/results

score $^{3}$

Experience

- Transition preparation of

youth $(\uparrow) \bigcirc$

- Transition

planning $(\uparrow) \bigcirc$

- Transfer of care $(\uparrow) \square$

A feasibility and

Type 1 diabetes Manitoba, A navigator Uncontrolled

Attendance/

Canada facilitated referrals cohort study

connecting

patients to family

physicians and

other support

when requested.

the Maestro Project-

community-based

administrative support

and systems navigation

service. Patients were

participation rate

- 373 Of the 473

(78.9\%) $\square$

- Project referrals and community

connections

contacted biannually to

( $) \square$

- Of the 373

participants, 127

requested 230

community

contacts for

assistance to

access care,

education or

optometry

services $\square$

- 34 Contact

numbers given

for family

physician care $\square$

- 121 Contacts to

reconnect with

diabetes

education and

counselling

services $\square$

- 203 Requests for

more information/ support $\square$ 
improvement in the development of a transition policy, transitioning youth registry, transition preparedness, transition planning, transfer of care and transition documentation. ${ }^{28}$ The study was set in primary care centres, each of which participated in a 2-year learning collaborative and had a team consisting of a lead physician, care coordinator focused on transition (ie, nurse, social worker) and consumer (parent/caregiver or young adult). ${ }^{34}$ Teams attended five $1 \frac{1}{2}$-day learning and coaching sessions. Sites were provided with financial support to cover staff time and patient participation. Each health centre evaluated their practice on the Core Elements using the Health Care Transition Index, and rated themselves in the six categories using a combination of narrative descriptions and raw scoring. The evaluation was completed three times over the course of the study.

The Maestro Project based in Manitoba, Canada is a navigation service to help young adults aged $18-30$ years with type 1 diabetes transfer to adult healthcare services. ${ }^{35}$ The Maestro Project maintains a database of 473 young adults living in Manitoba, north-western Ontario, and south-eastern Saskatchewan. The term 'Maestro' refers to an administrative project coordinator who provides support (via phone, email, newsletters or drop-in group sessions), identifies barriers to accessing healthcare services and connects young adults with specialty care (ie, general internists, adult endocrinologists), diabetes educators and counsellors, and family physicians. The Maestro also works with community centres involved in diabetes education in order to provide referrals and facilitate follow-up visits. This uncontrolled cohort study assessed the feasibility and acceptability of the Maestro Project by collecting participants' demographic information, self-reported medical outcomes at baseline and follow-ups, programme participation, project referrals and community connections using surveys administered biannually. Appropriate primary care providers were contacted by the Maestro to support diabetes care for youth transitioning to adult services.

\section{Triple Aim Framework domains and study outcomes Experience of care}

Experience of care was only assessed by the SCD retrospective cohort study, which used satisfaction surveys administered to patients, parents and healthcare providers. ${ }^{33}$ Hankins et $a l^{33}$ reported that all participants, parents and healthcare providers found the programme to be either 'helpful' or 'very helpful'. None of the other studies reviewed collected data on patient satisfaction or barriers to care.

\section{Population health}

Population health measures were used in two of the three studies reviewed. The McManus $e t a l^{34}$ time series comparative study of five health centres measured population health domain as adherence to care guidelines (using the AAP Clinical Report on Supporting the health care transition from adolescent to adulthood in the medical home) and found improvements in all six transition quality indicators. $^{28}$

\section{Cost}

Health service utilisation (participation/attendance) was measured in all studies. In the SCD retrospective cohort study, $41 \%$ of youth agreed to participate in the pilot programme, and $74 \%$ of participants completed their first appointment with an adult haematologist (compared with $33 \%$ of non-participants). ${ }^{33}$ Similarly, there was an increase in project referrals and community connections among Maestro Project participants. ${ }^{35}$

McManus $e t a l^{34}$ reported improvements in transfer of care scores after implementing the learning collaborative in participating health centres over 15 months. No studies assessed actual costs either as an outcome or an input associated with a transition programme or intervention.

\section{DISCUSSION}

This systematic review demonstrates that there is little empiric evidence to guide clinical practice or policy around the role of primary care providers in the transition of youth with chronic health conditions. We found only three published studies that evaluated any kind of primary care-specific intervention aimed at improving transition, only one of which was based in a primary care setting. The level of primary care engagement was limited. Two of the interventions included a case manager who facilitated appointments with a primary care provider during transition and therefore did not focus on primary care as the key target of intervention. Overall, none of the studies examined transition measures across all three Triple Aim Framework domains.

In a previous work, we have shown that very few jurisdictions have any health system strategy to address transition. ${ }^{40}$ However, in a joint clinical report published by the AAP, American Academy of Family Physicians (AAFP) and American College of Physicians (ACP), primary care providers and medical specialists were encouraged to adopt a transition planning algorithm for all youth within a medical home specific to their clinical setting. ${ }^{28}$ However, much of the emphasis in the transition literature (which most likely reflects clinical practice) focuses solely on specialist-to-specialist provider transition. ${ }^{41-48}$ This may reflect in part the de facto interest and leadership of the paediatric specialist community, as well as the generally fragmented nature of the primary to specialty care interface.

There is no gold standard for the organisation of care that will best meet transition needs for all youth with chronic conditions. A range of system-level approaches are most likely needed to improve the process. ${ }^{40} 424950$ The level and type of primary care involvement during transition should differ depending on the condition. ${ }^{4}$ For example, for some diseases such as uncomplicated 
epilepsy, the primary care provider may be the appropriate centre of medical management across transition, whereas for other more complex conditions such as cystic fibrosis, ongoing management will be centred at a specialised adult centre with primary care involved in a more supportive role. Youth with medical complexity may require a mixed strategy as they often have multiple specialist providers, but often care coordination occurs either in general paediatric clinics in children's hospitals, or by primary care paediatricians in a medical home. $^{25} 2851$ Many paediatric chronic diseases, however, may benefit from a shared-care model or a more staged approach where there is a phased transfer between paediatric subspecialists and primary care providers in a disease-specific way; however, we could not find any evidence to guide these types of approaches.

For any health system strategy around transition, primary care models for children will be an important consideration. In the UK, Australia and most parts of Canada, youth receive primary care from a general or family practitioner and are likely to continue with the same primary care provider as they move into adulthood. Such patients may still benefit from a process to help them improve readiness and increase responsibility for their care as they transition. ${ }^{4}{ }^{6}$ Other youth with chronic conditions whose primary care provider is a paediatrician, as opposed to a general practitioner, may experience a 'double hit' as they require transition from both primary and secondary or specialist care. We found no data to guide these 'double hit' transitions and whether they should occur simultaneously or in a sequential fashion. It is also striking that although the most common chronic conditions of childhood (eg, asthma) are managed almost exclusively by primary care providers, there are no studies evaluating primary care transition models in these populations in systems that rely heavily on paediatrician-led primary care, such as in most states in the USA.

\section{CONCLUSION}

This systematic review is limited by the lack of substantial evidence on the role of primary care in enhancing the quality of transition care for youth with chronic conditions. System strategies that account for differences in paediatric primary care providers and the levels of complexity of chronic conditions will be important. Other policy initiatives that seek to improve coordination of care, such as Accountable Care Organizations in the USA, may provide an opportunity to address the role of primary care in transition for youth across transition. It will be essential to establish consistent goals for primary care in transition in order to build an adequate body of literature to affect policy and practice. Future research and policy should focus on developing and evaluating coordinated transition interventions involving primary care to better integrate primary and specialised care.
Author affiliations

${ }^{1}$ Division of Paediatric Medicine, The Hospital for Sick Children, Toronto, Ontario, Canada

${ }^{2}$ Division of Adolescent Medicine, The Hospital for Sick Children, Toronto, Ontario, Canada

${ }^{3}$ Institute of Health Policy, Management, and Evaluation, University of Toronto, Toronto, Ontario, Canada

${ }^{4}$ Department of Paediatrics, University of Toronto, Toronto, Ontario, Canada

${ }^{5}$ Institute for Clinical Evaluative Sciences, Toronto, Ontario, Canada

Acknowledgements This project was completed with support from the Health System Performance Research Network (HSPRN).

Contributors AG obtained funding for the study. AG and EC conceived the study, supervised the activities of the research team and reviewed, edited and approved the manuscript. JB and AT conducted the systematic review and data abstraction for the study, drafted the initial manuscript and reviewed and approved the manuscript. CMH contributed ideas to the study design and reviewed and approved the manuscript.

Funding EC is supported as the 2015-2016 Commonwealth Fund/CFHI Harkness Fellow in Health Care Policy and Practice. The Commonwealth Fund is a private independent foundation based in New York City. AG is funded by a Canadian Institutes of Health Research Applied Chair in Child Health Services and Policy Research. This study was funded by the Health System Performance Research Network (HSPRN).

\section{Competing interests None.}

Provenance and peer review Not commissioned; externally peer reviewed. Data sharing statement No additional data are available.

Open Access This is an Open Access article distributed in accordance with the Creative Commons Attribution Non Commercial (CC BY-NC 4.0) license, which permits others to distribute, remix, adapt, build upon this work noncommercially, and license their derivative works on different terms, provided the original work is properly cited and the use is non-commercial. See: http:// creativecommons.org/licenses/by-nc/4.0/

\section{REFERENCES}

1. Pinzon J, Harvey J, Canadian Paediatric Society, Adolescent Health Committee. Care of adolescents with chronic conditions. Pediatr Child Health 2006;11:43-8.

2. Blum RW. Overview of transition issues for youth with disabilities. Paediatrician 1991;18:101-4.

3. Kaufman M, Pinzon J, Canadian Paediatrics Society, Adolescent Health Committee. Transition to adult care for youth with special health care needs. Pediatr Child Health 2007;12:785-93.

4. McPheeters M, Davis AM, Taylor JL, et al. Transition care for children with special health needs. Technical Brief No. 15 (Prepared by the Vanderbilt University Evidence-based Practice Center under Contract No. 290-2012-00009- I). AHRQ Publication No.14-EHC027EF. Rockville, MD: Agency for Healthcare Research and Quality, 2014. http://www.effectivehealthcare.ahrq.gov/reports/final.cfm (accessed 24 Feb 2015).

5. Reiss J. Health care transition for emerging adults with chronic health conditions and disabilities. Pediatr Ann 2012;41: 429-35.

6. Stinson J, Kohut SA, Spiegel L, et al. A systematic review of transition readiness and transfer satisfaction measures for adolescents with chronic illness. Int J Adolesc Med Health 2014;26:159-74.

7. Stoeck PA, Cheng N, Berry AJ, et al. Health care transition counseling for youth with special health care needs. Am Fam Physician 2012;86:1024.

8. McManus MA, Pollack LR, Cooley WC, et al. Current status of transition preparation among youth with special needs in the United States. Paediatrics 2013;131:1090-7.

9. Toomey SL, Chien AT, Elliott MN, et al. Disparities in unmet need for care coordination: the National Survey of Children's Health. Paediatrics 2013;131:217-24.

10. Okumura MJ, Heisler M, Davis MM, et al. Comfort of general internists and general paediatricians in providing care for young adults with chronic illnesses of childhood. J Gen Intern Med 2008;23:1621-7. 
11. Peter NG, Forke CM, Ginsburg KR, et al. Transition from paediatric to adult care: internists' perspectives. Paediatrics 2009;123:417-23.

12. Patel MS, O'Hare K. Residency training in transition of youth with childhood-onset chronic disease. Paediatrics 2010;126(Suppl 3): S190-3.

13. Amaria K, Stinson J, Cullen-Dean G, et al. Tools for addressing systems issues in transition. Healthc Q 2011:14(Spec No 3):72-6.

14. Scal P. Improving health care transition services: Just Grow Up, Will You Please. JAMA Pedatr 2016;170:197-9.

15. White $\mathrm{PH}, \mathrm{McManus} \mathrm{MA}, \mathrm{McAllister} \mathrm{JW}$, et al. A primary care quality improvement approach to health care transition. Pediatr Ann 2012;41:e1-7.

16. Lotstein DS, McPherson M, Strickland B, et al. Transition planning for youth with special health care needs: results from the National Survey of Children with Special Health Care Needs. Paediatrics 2005;115:1562-8

17. Hazel E, Zhang X, Duffy CM, et al. High rates of unsuccessful transfer to adult care among young adults with juvenile idiopathic arthritis. Pediatr Rheumatol Online J 2010;8:2.

18. Nakhla M, Daneman D, To T, et al. Transition to adult care for youths with diabetes mellitus: findings from a Universal Health Care System. Paediatrics 2009;124:e1134-41.

19. Reid GJ, Irvine MJ, McCrindle BW, et al. Prevalence and correlates of successful transfer from paediatric to adult health care among a cohort of young adults with complex congenital heart defects. Paediatrics 2004:113(Pt 1):e197-205.

20. Tomlinson P, Sugarman ID. Complications with shunts in adults with spina bifida. BMJ 1995;311:286-7.

21. Watson AR. Non-compliance and transfer from paediatric to adult transplant unit. Pediatr Nephrol 2000;14:469-72.

22. American Academy of Paediatrics, American Academy of Family Physicians, American College of Physicians-American Society of Internal Medicine. A consensus statement on health care transitions for young adults with special health care needs. Paediatrics 2002;110(Pt 2):1304-6.

23. Farre A, Wood V, McDonagh JE, et al. Transition Collaborative Group. Health professionals' and managers' definitions of developmentally appropriate healthcare for young people: conceptual dimensions and embedded controversies. Arch Dis Child 2016. Published Online First: 4 Mar 2016.

24. Scal P. Transition for youth with chronic conditions: primary care physicians' approaches. Paediatrics 2002;110(Pt 2):1315-21.

25. Crowley R, Wolfe I, Lock K, et al. Improving the transition between paediatric and adult healthcare: a systematic review. Arch Dis Child 2011:96:548-53.

26. Grant C, Pan J. A comparison of five transition programmes for youth with chronic illness in Canada. Child Care Health Dev 2011;37:815-20.

27. Blum RW, Garell D, Hodgman $\mathrm{CH}$, et al. Transition from child-centered to adult health-care systems for adolescents with chronic conditions: a position paper for the Society for Adolescent Medicine. J Adolsc Health 1993;14:570-6.

28. Cooley WC, Sagerman PJ, American Academy of Paediatrics; American Academy of Family Physicians; American College of Physicians; Transitions Clinical Report Authoring Group. Supporting the health care transition from adolescence to adulthood in the medical home. Paediatrics 2011:128:182-200.

29. Whitehouse SR, Rabinovitz SJ, Paone M, et al. ON TRACTransitioning responsibly to adult care. A collaborative provincial initiative to address transition from grass roots engagement to health service policy. J Adolsc Health 2015;56:S35.

30. Prior M, McManus M, White P, et al. Measuring the "Triple Aim" in transition care: a systematic review. Paediatrics 2014;134:e1648-61.

31. Stiefel M, Nolan K. A guide to measuring the Triple Aim: population health, experience of care, and per capita cost. IHI Innovation Series white paper. Cambridge, MA: Institute for Healthcare Improvement, 2012.
32. Hawker S, Payne S, Kerr C, et al. Appraising the evidence: reviewing disparate data systematically. Qual Health Res 2002;12:1284-99.

33. Hankins JS, Osarogiagbon R, Adams-graves $\mathrm{P}$, et al. A transition pilot program for adolescents with sickle cell disease. J Ped Healthcare 2012;26:e45-9.

34. McManus $\mathrm{M}$, White $\mathrm{P}$, Barbour $\mathrm{A}$, et al. Pediatric to adult transition: a quality improvement model for primary care. $J$ Adol Health 2015;56:73-8.

35. Van Wallegham N, Macdonald CA, Dean HJ. Building connections for young adults with type 1 diabetes mellitus in Manitoba: feasibility and acceptability of a transition initiative. Chron Dis Can 2006;27:130-4

36. Overholser L, Greffe B, Garrington T, et al. Implementation and evaluation of an innovative childhood cancer survivor follow-up clinic: Tactic. Conference abstract. J General Internal Med 2012;27: S526.

37. Kingsnorth S, Proulx M, Tsybina I, et al. Integrating a new model of transition care into real-life systems: issues in implementing the LIFEspan model. Conference proceedings. Dev Med Child Neurol 2012;54:20.

38. Wallis EM, Salek EC, Steinway C, et al. Transition from paediatric to adult healthcare for youth with complex chronic conditions: a primary care pilot study. Conference abstract. J Adol Health 2015;56(2): S125.

39. Dorsey D, Mayes S, Johnson A, et al. Transition for patients with sickle cell disease: is it possible to have a successful transition experience in communities without access to adult sickle cell programs? Conference abstract. Am J Hematol 2009;84(8): E156.

40. Moore Hepburn C, Cohen E, Bhawra J, et al. Health system strategies supporting transition to adult care. Arch Dis Child 2015;100:559-64.

41. Pinzon JL, Jacobson K, Reiss J. Say goodbye and say hello: the transition from pediatric to adult gastroenterology. Can J Gastroenterol 2004;18:735-42.

42. Robertson L. When should young people with chronic rheumatic disease move from paediatric to adult-centred care? Best Pract Res Clin Rheumatol 2006;20:387-97.

43. Callahan ST, Winitzer RF, Keenan P. Transition from paediatric to adult-oriented health care: a challenge for patients with chronic disease. Curr Opin Pediatr 2001:13:310-16.

44. Eleftheriou D, Isenberg DA, Wedderburn LR, et al. The coming of age of adolescent rheumatology. Nat Rev Rheumatol 2014;10:187-93.

45. Lyons SK, Libman IM, Sperling MA. Diabetes in the adolescent: transitional issues. J Clin Endocrinol Metab 2013;98:4639-45.

46. Young S, Murphy CM, Coghill D. Avoiding the 'twilight zone': recommendations for the transition of services from adolescence to adulthood for young people with ADHD. BMC Psychiatry 2011;11:174

47. Mennito SH, Clark JK. Transition medicine: a review of current theory and practice. South Med J 2010;103:339-42.

48. Tucker LB, Cabral DA. Transition of the adolescent patient with rheumatic disease: issues to consider. Pediatr Clin North Am 2005;52:641-52.

49. Herzer M, Goebel J, Cortina S. Transitioning cognitively impaired young patients with special health needs to adult oriented care: collaboration between medical providers and paediatric psychologists. Curr Opin Pediatr 2010;22:668-72.

50. Webb N, Harden $\mathrm{P}$, Lewis $\mathrm{C}$, et al. Building consensus on transition of transplant patients from paediatric to adult healthcare. Arch Dis Child 2010;95:606-11.

51. Cohen E, Kuo DZ, Agrawal R, et al. Children with medical complexity: an emerging population for clinical and research initiatives. Paediatrics 2011:127:529-38. 